\title{
Robotic device for automation of the control of the status of cables in the mining industry
}

\author{
Gasan Saypulaev*, Igor Merkuryev, and Polina Skulova \\ National Research University “MPEI”, Dept. of Robotics, Mechatronics, Dynamics and Machine Strength, 14, Krasnokazarmennaya, \\ Moscow, 111250, Russia
}

\begin{abstract}
In this work, we propose a design of a robotic device designed to automate the process of nondestructive testing of wire rope equipment. A schematic hydraulic diagram of a robotic device with a description of the logic of work of the system is given. A mathematical model of the dynamics of the system is obtained using the Lagrange formalism. An algorithm for evaluating the control moments necessary for the implementation of programmed motion has been developed. Numerical simulation of the motion corresponding to the mode of approaching the magnetic flaw detector heads to the steel rope has been carried out. The graphs of the traction-speed characteristics and load diagrams of the drives providing the programmed movement of the links of the pantograph mechanism are presented. The results presented in this paper can be used to calculate the parameters of the power section of the electro-hydraulic drives included in the robotic device.
\end{abstract}

\section{Introduction}

\subsection{Formulation of the problem}

At present, the tasks associated with non-destructive testing technologies are becoming especially relevant due to the widespread use of modern science and technology. The main methods, prospects and trends of non-destructive testing of metals can be found in the review [1]. Many tasks in this area relate to the problems of carrying out non-destructive testing of wire rope equipment. The need to diagnose the condition of the ropes is due to the deterioration of the integrity of the steel ropes due to corrosion, wear and tear, which can lead to disasters.

New and more accurate methods of non-destructive testing [2-5] of cable equipment are already known and continue to be actively developed. These methods are based on the measurement of the cross-sectional area using magnetic flaw detectors, which makes it possible to estimate the decrease in the cross-sectional area of steel ropes over time. Because of such non-destructive testing methods, it is possible not only to assess the current state and integrity of steel ropes, but also to predict the change in their state after a certain period of time [6].

In addition to the problems associated with the development of methods and algorithms for nondestructive testing, many published works are devoted to the problems of development and improvement of devices for assessing defects in steel ropes [7-9], as well

\footnotetext{
* Corresponding author: bot05_00@mail.ru
}

as the automation of the procedure for non-destructive testing [10-12].

Thus, in this paper, we consider the problem of developing a robotic device for periodic monitoring of the technical condition of the cables of mine lifting equipment in hard-to-reach and dangerous places for personnel.

In order to increase labor productivity, reduce downtime and increase the safety of operation of the main equipment of the mine, the task of rational choice of the constructive kinematic and power scheme of the robot has been set.

\subsection{The design of the proposed robotic device}

An automatic robotic complex is a controlled multi-link device with target equipment for magnetic flaw detection to perform a predetermined set of technological tasks in difficult operating conditions without the direct participation of non-destructive testing specialists. As part of the robotic device, the INTROS-AUTO magnetic flaw detector of the INTRON PLUS company [13] is used, designed for non-destructive testing and technical diagnostics of steel ropes of mine, drilling rigs and other structures [14].

Periodic assessment of the degree of wear of the steel cable by measuring the cross-section of the cable along its length is carried out during the normal operation of the hoisting mine system. The robotic complex provides automatic approach of two magnetic heads of the flaw detector (Fig. 1) to the steel cable, which has the ability to arbitrarily move within the hatch, position these heads opposite each other and hold them in this position during 
measurements (Fig. 2). At the end of the measurements, the magnetic heads are automatically retracted from the cable and placed in the technological niches of the object.

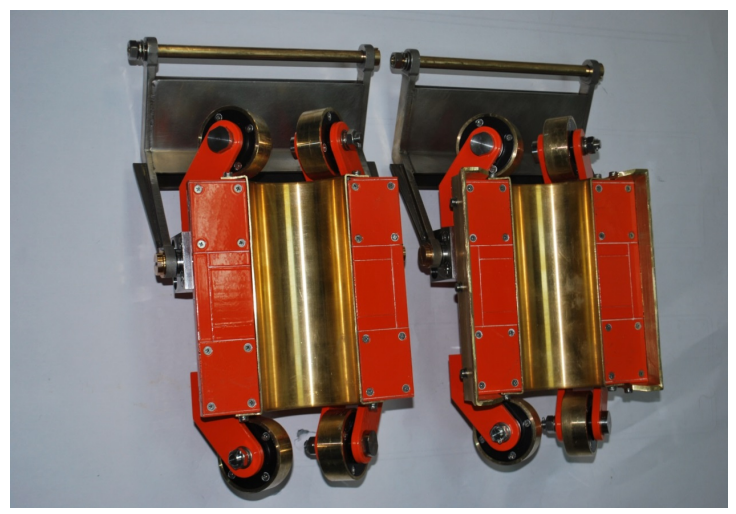

Fig. 1. Flaw detector magnetic heads with the roller system.

Taking into account the fact that the system operates at relatively low speeds in the modes of withdrawal and supply of cylindrical heads and significant loads arising from the disengagement of cylindrical heads, which must be carried out after the end of diagnostics, a hydraulic drive system of cyclic automation was chosen as a drive.

After connecting the cylindrical heads during operation in the diagnostic mode, the cable can move within the dimensions of the hatch due to various external disturbances. The proposed kinematic scheme, using a pantograph mechanism, allows automatic free synchronous movement of the links of the robotic device with the provision of a guaranteed gap between the surfaces of the head body and the cable.

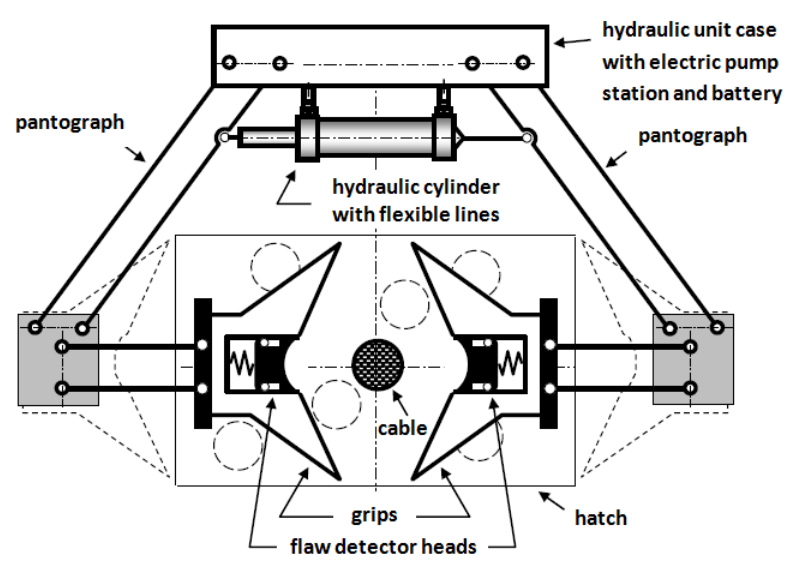

Fig. 2. Constructive scheme of the pantograph mechanism in the horizontal plane.

\subsection{Hydraulic system of the proposed robotic device}

A schematic hydraulic diagram of the power section of the proposed robotic device is shown in Fig. 3. Below we briefly describe the purpose of each element.

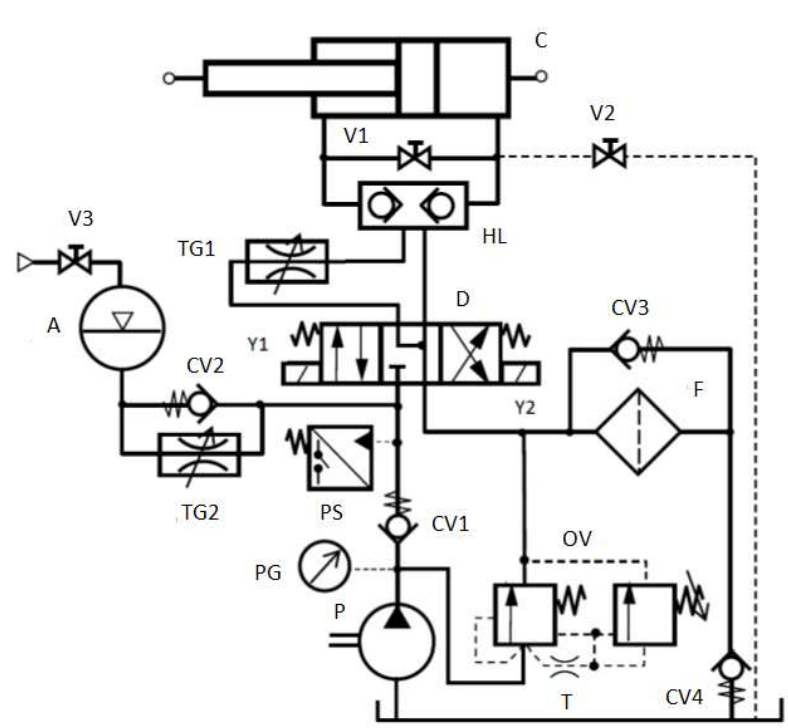

Fig. 3. Schematic hydraulic diagram of the robotic system.

The throttle governor TG1 is used to regulate the speed of movement of the output link of the cylinder (C) for the forward and reverse motion of the grippers. The double-sided hydraulic lock (HL) provides fixation of the position of the rod C. If necessary, it is possible to install a HL with thermal valves that exclude fluid compression.

The pneumohydraulic accumulator (A) provides a reverse movement of the rod $\mathrm{C}$ (divergence of the grippers).

The CV2 check valve is a charging one, and the TG2 throttle provides the emptying (discharging) of the A with a flow rate that gives the required speed of movement of the $\mathrm{C}$ rod. For reasons of compactness, reliability and a relatively short period of system operation, a constant flow pump (P) with an overflow valve was used when diagnosing the cable condition, bypassing the liquid through the hydrotreater $(\mathrm{F})$ into the open hydraulic tank (T). The CV3 check valve is used to protect the filter element $F$ from breakthrough in case of contamination. The $\mathrm{CV} 4$ check valve eliminates air leakage from the atmosphere by providing a back-up drain pressure. The directional valve (D) carries out the forward and reverse travel of the output link C (convergence and divergence of the grippers). Valves $\mathrm{V} 1, \mathrm{~V} 2$ are blocked in normal operation mode and open for "manual" movement of the C stem when the pump is off in order to service the system and routine maintenance. The V3 valve is used to charge the $\mathrm{A}$ at the manufacturer of this unit; during the operation of the system, the valve is blocked and sealed.

The presence of A allows one to optimize power consumption, improve thermal conditions and increase the reliability and survivability of the system, ensuring, in particular, the removal of the device blocks from the cable even in case of emergency power outage. For this purpose, the working volume of the A is calculated from the condition of ensuring the forward and reverse movement of the grippers. A pressure switch (PS) installed in the pressure line automatically turns on the driving electric motor (EM) of the pump when the 
pressure in the pressure line drops below the set value and turns off the EM when the required pressure is reached. In a normal or emergency situation (for example, in the event of a break in the power supply to the ED), the energy stored in the A is used for the return stroke of the $\mathrm{C}$ rod to remove the grippers with the magnetic heads of the flaw detector from the steel cable.

After describing the general logic of work and the design of the robotic complex, as the next stage of work, it is necessary to calculate the traction-speed characteristics of the system and assess the energy consumption required for the movement. For this purpose, it is necessary to construct a mathematical model of the dynamics of the robotic device.

\section{Dynamical model of the system}

\subsection{Kinematic scheme}

To compose the equations of the dynamics of the robotic device, we will use the Lagrange formalism [15], preliminarily introducing some designations on the kinematic scheme of the device (Fig. 4).

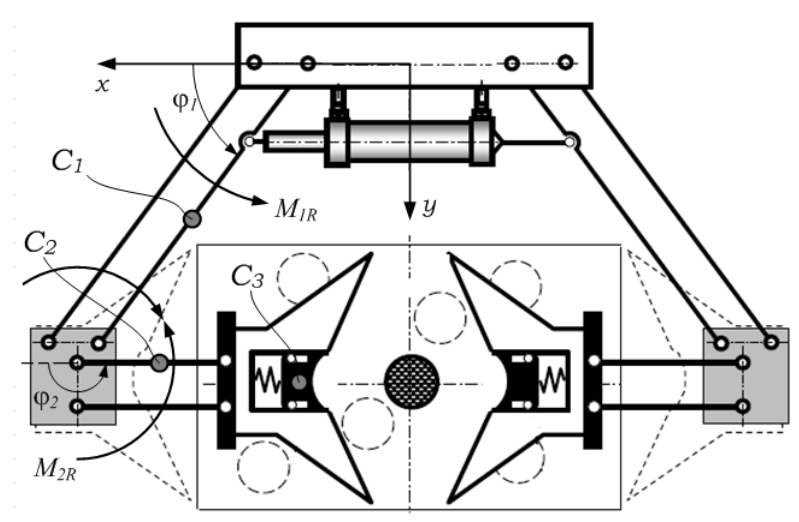

Fig. 4. Kinematic scheme of a robotic device.

The following designations are introduced on the scheme (Fig. 4):

-centers of mass of the first links of the mechanism $C_{1}$;

-centers of mass of the second links of the mechanism $C_{2}$;

-centers of mass of the cylindrical heads of the measuring device $C_{3}$;

-angle of rotation of the first link $\varphi_{1}$;

-angle of rotation of the second link $\varphi_{2}$;

-control torque $M_{1 R}$, driving the first link of the mechanism;

-control torque $M_{2 R}$, driving the second link of the mechanism;
All the angles of rotation of the links of the mechanism shown in Fig. 4 are counted from the horizontal axis in a counterclockwise direction.

\subsection{Equations of motion}

The Lagrange equations describing the dynamics of a system of bodies have the form:

$$
\frac{d}{d t}\left(\frac{\partial T}{\partial \dot{\mathbf{q}}}\right)-\frac{\partial T}{\partial \mathbf{q}}=-\frac{\partial \Pi}{\partial \mathbf{q}}-\frac{\partial \Phi}{\partial \dot{\mathbf{q}}}+Q_{M},
$$

where $\mathbf{q}=\left(\begin{array}{ll}\varphi_{1} & \varphi_{2}\end{array}\right)^{\mathrm{T}}$ is Lagrange coordinates vector; $\dot{\mathbf{q}}=\left(\begin{array}{ll}\dot{\varphi}_{1} & \dot{\varphi}_{2}\end{array}\right)^{\mathrm{T}}$ is generalized speeds; $T$ and $\Pi$ are kinetic and potential energies of the system $(\Pi=0$, since the movement of the mechanism occurs in a horizontal plane and the action of gravity does not affect the movement in the plane under consideration); $\Phi$ is Rayleigh dissipation function; $Q_{M}$ is generalized forces vector corresponding to control moments.

\subsubsection{Calculation of the inertial terms of the equations of motion}

The kinetic energy of the system has the form:

$$
T=\frac{1}{2}\left(m_{1} V_{C 1}^{2}+J_{1} \dot{\varphi}_{1}^{2}+m_{2} V_{C 2}^{2}+J_{2} \dot{\varphi}_{2}^{2}+m_{3} V_{C 3}^{2}\right),
$$

where $m_{1}, m_{2}, m_{3}-$ masses of the first and second links of the mechanism and the cylindrical head of the device; $J_{1}, J_{2}-$ moments of inertia of the first and second links of the mechanism; $V_{C 1}, V_{C 2}, V_{C 3}$ - modules of velocities of points $C_{1}, C_{2}, C_{3}$.

In accordance with the kinematic diagram (Fig. 3), the expressions for the projections of the velocities $V_{C 1}, V_{C 2}, V_{C 3}$ on the axes $x y$ can be written in the form:

$$
\begin{aligned}
& V_{C 1 x}=-\dot{\varphi}_{1} a_{1} \sin \varphi_{1}, \quad V_{C 1 y}=-\dot{\varphi}_{1} a_{1} \cos \varphi_{1}, \\
& V_{C 2 x}=-\dot{\varphi}_{1} l_{1} \sin \varphi_{1}-\dot{\varphi}_{2} a_{2} \sin \varphi_{2}, \\
& V_{C 2 y}=\dot{\varphi}_{1} l_{1} \cos \varphi_{1}+\dot{\varphi}_{2} a_{2} \cos \varphi_{2}, \\
& V_{C 3 x}=-\dot{\varphi}_{1} l_{1} \sin \varphi_{1}-\dot{\varphi}_{2} l_{2} \sin \varphi_{2}, \\
& V_{C 3 y}=\dot{\varphi}_{1} l_{1} \cos \varphi_{1}+\dot{\varphi}_{2} l_{2} \cos \varphi_{2} .
\end{aligned}
$$

Here $l_{1}, l_{2}$ are the lengths of the first and second links of the mechanism; $a_{1}, a_{2}$ are the distances to the centers of mass for the first and second links.

Following the Lagrange procedure, we calculate the left side of equations (1) taking into account expressions (2) and (3). In this case, we get: 


$$
\frac{d}{d t}\left(\frac{\partial T}{\partial \dot{\mathbf{q}}}\right)-\frac{\partial T}{\partial \mathbf{q}}=\mathbf{A}(\mathbf{q}) \ddot{\mathbf{q}}+\mathbf{F}(\mathbf{q}) \dot{\mathbf{q}}^{2}
$$

where the notation is introduced for the matrices of inertial and Coriolis forces:

$$
\begin{aligned}
& \mathbf{A}(\mathbf{q})=\left[\begin{array}{cc}
J_{1 R} & J_{12 R} \cos \left(\varphi_{1}-\varphi_{2}\right) \\
J_{12 R} \cos \left(\varphi_{1}-\varphi_{2}\right) & J_{2 R}
\end{array}\right], \\
& \mathbf{F}(\mathbf{q})=\left[\begin{array}{cc}
0 & J_{12 R} \sin \left(\varphi_{1}-\varphi_{2}\right) \\
-J_{12 R} \sin \left(\varphi_{1}-\varphi_{2}\right) & 0
\end{array}\right],
\end{aligned}
$$

vectors $\ddot{\mathbf{q}}=\left(\begin{array}{ll}\ddot{\varphi}_{1} & \ddot{\varphi}_{2}\end{array}\right)^{\mathrm{T}}, \quad \dot{\mathbf{q}}^{2}=\left(\begin{array}{ll}\dot{\varphi}_{1}^{2} & \dot{\varphi}_{2}^{2}\end{array}\right)^{\mathrm{T}}$ and reduced moments of inertia:

$$
\begin{aligned}
& J_{1 R}=J_{1}+m_{1} a_{1}^{2}+\left(m_{2}+m_{3}\right) l_{1}^{2}, \\
& J_{2 R}=J_{2}+m_{2} a_{2}^{2}+m_{3} l_{2}^{2}, \quad J_{12 R}=l_{1}\left(m_{2} a_{2}+m_{3} l_{2}\right) .
\end{aligned}
$$

\subsubsection{Calculation of generalized forces}

Now we write out the expressions for the dissipative function (due to the linearity of the viscous friction forces in the joints) and the generalized forces $Q_{M}$ :

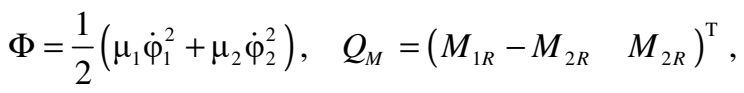

where $\mu_{1}, \mu_{2}$ - dissipation coefficients.

Then the right-hand side of the equations of motion (1), taking into account expressions (7), takes the form:

$$
Q_{M}-\frac{\partial \Phi}{\partial \dot{\mathbf{q}}}-\frac{\partial \Pi}{\partial \mathbf{q}}=\left[\begin{array}{c}
M_{1 R}-M_{2 R}-\mu_{1} \dot{\varphi}_{1} \\
M_{2 R}-\mu_{2} \dot{\varphi}_{2}
\end{array}\right] .
$$

\subsubsection{Equations of motion of the mechanism in scalar form}

Substituting expressions (4), (5) and (8) into Lagrange equations (1), we obtain a system of two differential equations describing the nonlinear dynamics of the considered model of the pantograph mechanism in the form:

$$
\begin{array}{r}
J_{1 R} \ddot{\varphi}_{1}+J_{12 R}\left[\ddot{\varphi}_{2} \cos \left(\varphi_{1}-\varphi_{2}\right)+\dot{\varphi}_{2}^{2} \sin \left(\varphi_{1}-\varphi_{2}\right)\right]= \\
=M_{1 R}-M_{2 R}-\mu_{1} \dot{\varphi}_{1}, \\
J_{2 R} \ddot{\varphi}_{2}+J_{12 R}\left[\ddot{\varphi}_{1} \cos \left(\varphi_{1}-\varphi_{2}\right)-\dot{\varphi}_{1}^{2} \sin \left(\varphi_{1}-\varphi_{2}\right)\right]= \\
=M_{2 R}-\mu_{2} \dot{\varphi}_{2} .
\end{array}
$$

The obtained equations of motion (9) can be used to estimate the energy consumption, which is necessary for the implementation of the programmed motion.

\subsection{Programmed motion and an algorithm for evaluating control actions}

\subsubsection{Algorithm for evaluating control actions}

To estimate the control torques, we will use the following algorithm:

1. We set the programmed movement of the links - the dependence of the angles $\varphi_{1}^{d}(t), \varphi_{2}^{d}(t)$;

2. We calculate the angular velocities $\dot{\varphi}_{1}^{d}(t), \dot{\varphi}_{2}^{d}(t)$ and angular accelerations $\ddot{\varphi}_{1}^{d}(t), \ddot{\varphi}_{2}^{d}(t)$;

3. From the second equation of dynamics (9) when substituting the programmed motion, we calculate the control torque $M_{2 R}^{d}(t)$ on the programmed motion;

4. From the first equation of dynamics, when substituting the programmed motion and the torque $M_{2 R}^{d}(t)$, we calculate the control torque $M_{1 R}^{d}(t)$ on the programmed motion.

\subsubsection{A special case of programmed motion}

Assuming to use a robotic device for approaching (connecting) and withdrawing (disconnecting) the magnetic heads of the flaw detector as a programmed motion, consider the laws of a particular case of motion when the second link of the mechanism maintains a constant orientation (i.e. $\varphi_{2}(t)=180^{\circ}, \quad \dot{\varphi}_{2}(t)=0$, $\left.\ddot{\varphi}_{2}(t)=0\right)$ along the axis $x$.

With such a motion, the equations of dynamics are reduced to one nonlinear second-order equation and the expression for the torque $M_{2 R}$ :

$$
\begin{aligned}
& J_{1 R} \ddot{\varphi}_{1}+J_{12 R}\left(-\ddot{\varphi}_{1} \cos \varphi_{1}+\dot{\varphi}_{1}^{2} \sin \varphi_{1}\right)=M_{1 R}-\mu_{1} \dot{\varphi}_{1}, \\
& M_{2 R}=J_{12 R}\left(-\ddot{\varphi}_{1} \cos \varphi_{1}+\dot{\varphi}_{1}^{2} \sin \varphi_{1}\right) .
\end{aligned}
$$

When the magnetic heads are brought in, the angle changes from the initial value $\varphi_{1}(0)$ (corresponding to the disconnected flaw detector heads retracted into production niches) to the final value $\varphi_{1}(T)$ (corresponding to the moment when the magnetic heads are connected when the wire rope is covered). In this case, as the law of variation of the angle, we take the "smooth" curve of dependence described by the formula:

$$
\varphi_{i}(t)=\varphi_{i}(0)+\left(\varphi_{i}(T)-\varphi_{i}(0)\right)\left[\frac{t}{T}-\frac{1}{2 \pi} \sin \left(\frac{2 \pi t}{T}\right)\right]
$$

Here $T$ - period of movement.

Due to the symmetry of the operations of approaching and withdrawing the magnetic heads to the rope, we will further simulate only one of these 
movements. As an example, we consider the convergence mode.

\section{Results of numerical simulation}

As an example, we consider the following numerical parameters of the mathematical model:

$$
\begin{aligned}
& J_{1 R}=26.6667 \mathrm{~kg} \cdot \mathrm{m}^{2}, \quad J_{2 R}=3.4667 \mathrm{~kg} \cdot \mathrm{m}^{2} \\
& J_{12 R}=9.0 \mathrm{~kg} \cdot \mathrm{m}^{2}, \quad \mu_{1}=\mu_{1}=1.2 \cdot 10^{-3} \mathrm{~N} \cdot \mathrm{m} \cdot \mathrm{s}
\end{aligned}
$$

In this case, we will take the following values of the parameters characterizing the programmed motion:

$$
\varphi_{1}(0)=30^{\circ}, \quad \varphi_{1}(T)=75^{\circ}, \quad T=3 \mathrm{~s} .
$$

Let us carry out numerical modeling of equations (10) in accordance with the algorithm given in Section 2.3.1 of this article, using the symbolic mathematics package Wolfram Mathematica [16].

Based on the simulation results, we will construct graphs of time dependences for generalized coordinates (Fig. 5), velocities (Fig. 6) and accelerations (Fig. 7), as well as control torques (Fig. 8).

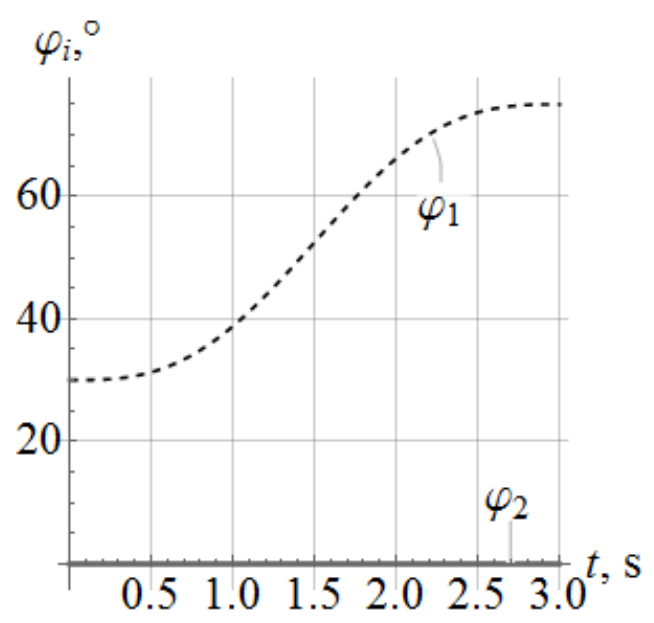

Fig. 5. Angles of rotation of the links of the robotic complex when bringing the flaw detector heads to the rope.

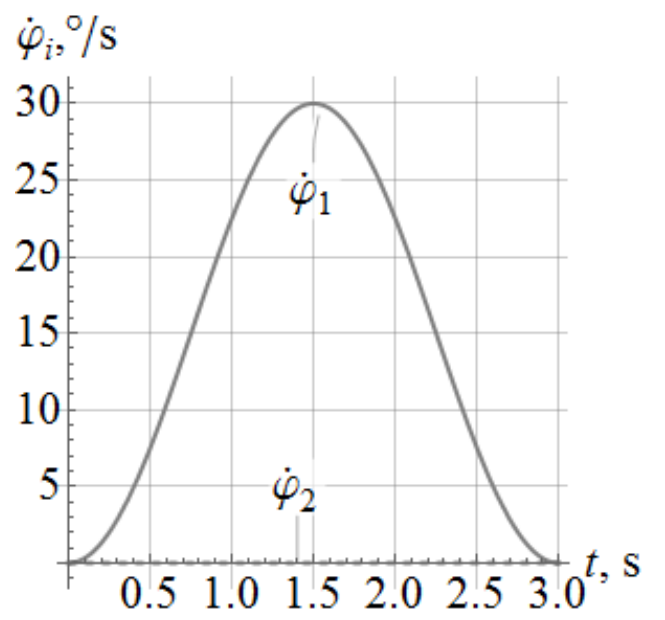

Fig. 6. Angles of rotation of the links of the robotic complex when bringing the flaw detector heads to the rope.

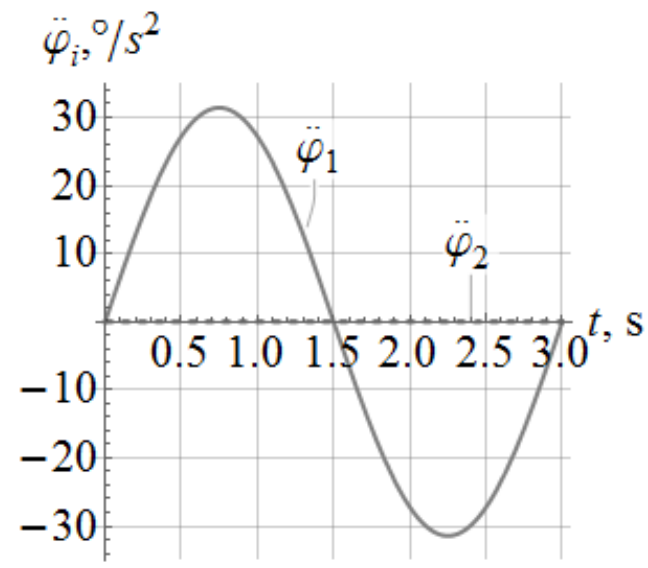

Fig. 7. Angular accelerations of the links of the robotic complex when bringing the flaw detector heads to the rope.

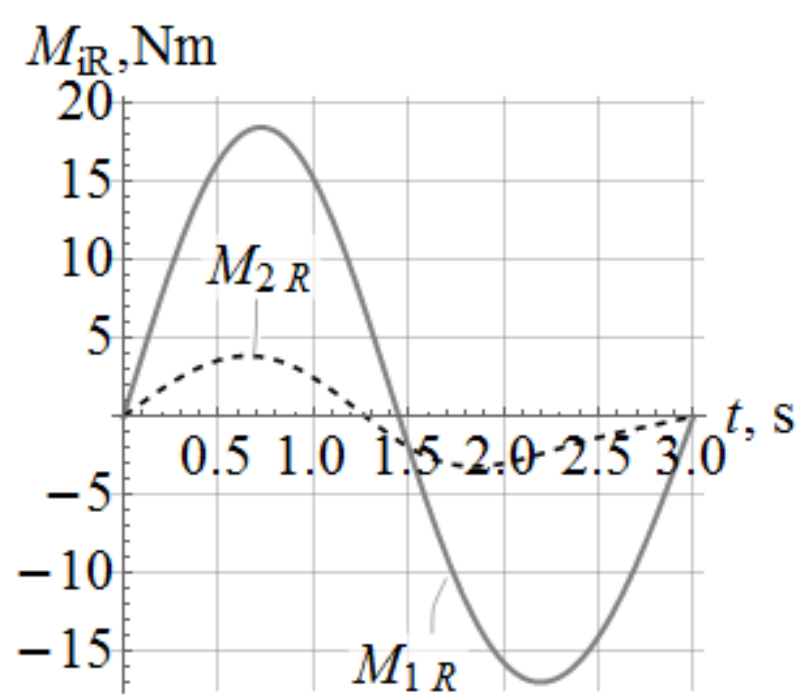

Fig. 8. Control torques in the hinges of the pantograph mechanism when bringing the flaw detector heads to the rope. 
According to the graph shown in Fig. 8, it is possible to estimate the maximum values of the moments that the drives must develop. The specified values can be used to calculate the power section of the drive system.

\section{Results and Discussion}

To demonstrate the traction-speed characteristics of the drive, we give the load diagrams in Fig. 9. The specified diagram is the initial data for calculating the electrohydraulic drive.

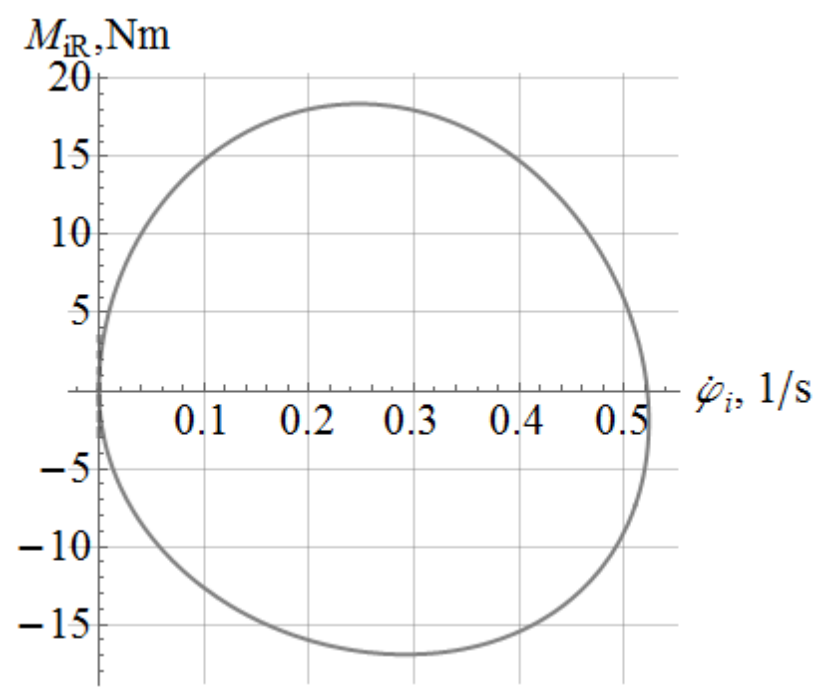

Fig. 9. Load diagram for the drive of the first link of the pantograph mechanism when approaching the flaw detector heads to the rope.

\section{Conclusion}

In this work, the following results are obtained:

- the design of a robotic device for automating the cable diagnostics process has been proposed;

- a mathematical model of the dynamics of the system has been built, which can be used to calculate the power section of an electrohydraulic drive that provides the required programmed motion;

- an algorithm has been developed for evaluating the control torques necessary for working out the programmed motion.

- numerical simulation was carried out for the programmed motion corresponding to bringing the heads of the magnetic flaw detector to the steel rope.

\section{Acknowledgements}

The investigation was carried out within the framework of the project "Development of a prototype of a new autonomous mobile robot for solving problems of monitoring the technical condition of cable equipment" with the support of a grant from NRU "MPEI" for implementation of scientific research programs "Energy", "Electronics, Radio Engineering and IT", and "Industry 4.0, Technologies for Industry and Robotics in 2020-2022".

\section{References}

1. X. Mao, Y. Zhao and T. Xiao, 2018 IEEE 4th Information Technology and Mechatronics Engineering Conference (ITOEC), pp. 926-929 (2018).

2. T. Moriya, M. Sugawara and K. Tsukada, Oceans '04 MTS/IEEE Techno-Ocean '04, pp. 19041909 (2004).

3. V. V. Sukhorukov, Materials Evaluation, pp. 1645-1651 (2016).

4. V. V. Sukhorukov, Materials Evaluation, pp. 26-31 (2013).

5. D. Slesarev and A. Semenov, Proceedings of the 12th ECNDT 2018 - European conference on Non-Destructive Testing, pp. 1-3 (2018)

6. V. V. Sukhorukov, Proceedings of the 12-th International Conference of the Slovenian Society for Non-Destructive Testing, pp. 389-402 (2013).

7. X. Yan, D. Zhang and H. Wang, IEEE Transactions on Instrumentation and Measurement, 69, 4487-4495 (2019).

8. S. Pan, D. Zhang and H. Wang, 2019 IEEE 3rd Advanced Information Management, Communicates, Electronic and Automation Control Conference (IMCEC), pp. 180-185 (2019).

9. L. Ren, Z. Liu, H. Wang, J. Zhou and H. Tang, 2019 IEEE 4th Advanced Information Technology, Electronic and Automation Control Conference (IAEAC), pp. 1037-1041 (2019).

10. K. Sakai, K. Morita, Y. Haga, T. Kiwa, K. Inoue and K. Tsukada, IEEE Transactions on Magnetics, 51, 1-3 (2015).

11. K. Myakushev, D. Slesarev and D. Sukhorukov, Proceedings of the 12th ECNDT 2018 - European conference on Non-Destructive Testing, pp. 1-3 (2018)

12. D. Slesarev, Signal Processing, 63, 146150 (2021).

13. INTRON PLUS. Non-destructive testing and technical diagnostics, Retrieved from: https://www.intron.ru/ru/

14. M. M. Matlin, A. I. Mozgunova, E. N. Kazankina, V. A. Kazankin, Methods for non-destructive testing of the strength properties of machine parts, Innovative mechanical engineering, Moscow, 2019, 247 p. (in Russian) 
15. A. P. Markeev, Theoretical mechanics, Regular and chaotic mechanics, Moscow, 2007, 592 p. (in Russian)

16. WOLFRAM MATHEMATICA Retrieved from: https://www.wolfram.com/mathematica $\underline{1}$ 